\title{
New conformable fractional integrals of Ostrowski type using new generalized $(s, m, \varphi)$-preinvex mappings
}

\author{
Artion Kashuri ${ }^{1, a}$, Rozana Liko ${ }^{1, b}$
}

\begin{abstract}
In the present paper, the notion of new generalized $(s, m, \varphi)$-preinvex mapping is introduced and some new integral inequalities for the left-hand side of Gauss-Jacobi type quadrature formula involving new generalized $(s, m, \varphi)$-preinvex mappings along with beta function are given. Moreover, some generalizations of Ostrowski type inequalities for new generalized $(s, m, \varphi)$-preinvex mappings via conformable fractional integrals are established. At the end, some applications to special means are given.

2010 Mathematics Subject Classification. Primary: 26A51; Secondary: 26A33, 26D07, 26D10, 26D15. Key words and phrases. Ostrowski type inequality, Hölder's inequality, Power mean inequality, RiemannLiouville fractional integrals, Conformable fractional integrals, $s$-convex function in the second sense, $m$-invex, $P$-function.
\end{abstract}

\section{Introduction}

The following notations are used throughout this paper. We use $I$ to denote an interval on the real line $\mathbb{R}=$ $(-\infty,+\infty)$ and $I^{\circ}$ to denote the interior of $I$. The nonnegative real numbers are denoted by $\mathbb{R}_{\circ}=[0,+\infty)$. The set of integrable functions on the interval $[a, b]$ is denoted by $L_{1}[a, b]$.

The following result is known in the literature as the Ostrowski inequality, see [22] and the references cited therein, which gives an upper bound for the approximation of the integral average $\frac{1}{b-a} \int_{a}^{b} f(t) d t$ by the value $f(x)$ at point $x \in[a, b]$.

Received June 06, 2017 - Accepted May 22, 2018, published online in July 2018.

(C)The Author(s) 2017. This article is published with open access by Sidi Mohamed Ben Abdallah University.

${ }^{1}$ Department of Mathematics, Faculty of Technical Science, University Ismail Qemali, Vlora, Albania

${ }^{a}$ e-mail: artionkashuri@gmail.com

${ }^{b}$ e-mail: rozanaliko86@gmail.com 
Theorem 1.1. Let $f: I \longrightarrow \mathbb{R}$, where $I \subseteq \mathbb{R}$, be a mapping differentiable on $I^{\circ}$, and let $a, b \in I^{\circ}$ with $a<b$. If $\left|f^{\prime}(x)\right| \leq M$ for all $x \in[a, b]$, then

$$
\left|f(x)-\frac{1}{b-a} \int_{a}^{b} f(t) d t\right| \leq M(b-a)\left[\frac{1}{4}+\frac{\left(x-\frac{a+b}{2}\right)^{2}}{(b-a)^{2}}\right], \quad \forall x \in[a, b] .
$$

For other recent results concerning Ostrowski type inequalities (see [[16],[22],[28], [33]]) and the references cited therein.

Fractional calculus [23] and the references cited therein, was introduced at the end of the nineteenth century by Liouville and Riemann, the subject of which has become a rapidly growing area and has found applications in diverse fields ranging from physical sciences and engineering to biological sciences and economics.

Definition 1.1. Let $f \in L_{1}[a, b]$. The Riemann-Liouville integrals $J_{a+}^{\alpha} f$ and $J_{b-}^{\alpha} f$ of order $\alpha>0$ with $a \geq 0$ are defined by

$$
J_{a+}^{\alpha} f(x)=\frac{1}{\Gamma(\alpha)} \int_{a}^{x}(x-t)^{\alpha-1} f(t) d t, \quad x>a
$$

and

$$
J_{b-}^{\alpha} f(x)=\frac{1}{\Gamma(\alpha)} \int_{x}^{b}(t-x)^{\alpha-1} f(t) d t, \quad b>x,
$$

where $\Gamma(\alpha)=\int_{0}^{+\infty} e^{-u} u^{\alpha-1} d u$. Here $J_{a+}^{0} f(x)=J_{b-}^{0} f(x)=f(x)$.

In the case of $\alpha=1$, the fractional integral reduces to the classical integral.

Due to the wide application of fractional integrals, some authors extended to study fractional Hermite-Hadamard and Ostrowski type inequalities for functions of different classes (see [[4]-[8],[10]-[18],,[20],[22],[23],[26]-[28],[30],[31],[33],,[35]] and the references cited therein.

The following definitions will be used in the sequel.

Definition 1.2. The Euler beta function is defined for $a, b>0$ as

$$
\beta(a, b)=\int_{0}^{1} t^{a-1}(1-t)^{b-1} d t=\frac{\Gamma(a) \Gamma(b)}{\Gamma(a+b)} .
$$

Definition 1.3. The incomplete beta function is defined for $a, b>0$ as

$$
\beta_{x}(a, b)=\int_{0}^{x} t^{a-1}(1-t)^{b-1} d t, \quad 0<x \leq 1 .
$$

For $x=1$, the incomplete beta function coincides with the complete beta function.

In the following, we give some definitions and properties of conformable fractional integrals which help to obtain main identity and results. Recently, some authors, started to study on conformable fractional integrals. In [19], Khalil et al. defined the fractional integral of order $0<\alpha \leq 1$ only. In [1], Abdeljawad gave the definition of left and right conformable fractional integrals of any order $\alpha>0$.

Definition 1.4. Let $\alpha \in(n, n+1]$ and set $\beta=\alpha-n$, then the left conformable fractional integral starting at a is defined by

$$
\left(I_{\alpha}^{a} f\right)(t)=\frac{1}{n !} \int_{a}^{t}(t-x)^{n}(x-a)^{\beta-1} f(x) d x .
$$

Analogously, the right conformable fractional integral is defined by

$$
\left({ }^{b} I_{\alpha} f\right)(t)=\frac{1}{n !} \int_{t}^{b}(x-t)^{n}(b-x)^{\beta-1} f(x) d x .
$$


Notice that if $\alpha=n+1$, then $\beta=\alpha-n=n+1-n=1$, where $n=0,1,2, \ldots$, and hence $\left(I_{\alpha}^{a} f\right)(t)=\left(J_{n+1}^{a} f\right)(t)$.

Set et al. in [26] established a generalization of Hermite-Hadamard type inequality for s-convex functions and gave some remarks to show the relationships with the classical and Riemann-Liouville fractional integrals inequality by using the given properties of conformable fractional integrals.

Theorem 1.2. Let $f:[a, b] \longrightarrow \mathbb{R}$ be a function with $0 \leq a<b, s \in(0,1]$, and $f \in L_{1}[a, b]$. If $f$ is a convex function on $[a, b]$, then the following inequalities for conformable fractional integrals hold:

$$
\begin{aligned}
& \frac{\Gamma(\alpha-n)}{\Gamma(\alpha+1)} f\left(\frac{a+b}{2}\right) \leq \frac{1}{2^{s}(b-a)^{\alpha}}\left[\left(I_{\alpha}^{a} f\right)(b)+\left({ }^{b} I_{\alpha} f\right)(a)\right] \\
& \leq\left[\frac{\beta(n+s+1, \alpha-n)+\beta(n+1, \alpha-n+s)}{n !}\right] \frac{f(a)+f(b)}{2^{s}},
\end{aligned}
$$

with $\alpha \in(n, n+1], n \in \mathbb{N}, n=0,1,2, \ldots$, where $\Gamma$ is Euler gamma function.

Also Set et al. established some results for some kind of inequalities via conformable fractional integrals (see [[27]$[29],[31]])$.

Now, let us recall some definitions of various convex functions.

Definition 1.5. [6] A non-negative function $f: I \subseteq \mathbb{R} \longrightarrow \mathbb{R}_{\circ}$ is said to be P-function, if

$$
f(t x+(1-t) y) \leq f(x)+f(y), \quad \forall x, y \in I, t \in[0,1]
$$

Definition 1.6. [9] A function $f: \mathbb{R}_{\circ} \longrightarrow \mathbb{R}$ is said to be s-convex in the second sense, if

$$
f(\lambda x+(1-\lambda) y) \leq \lambda^{s} f(x)+(1-\lambda)^{s} f(y)
$$

for all $x, y \in \mathbb{R}_{\circ}, \lambda \in[0,1]$ and $s \in(0,1]$.

It is clear that a 1-convex function must be convex on $\mathbb{R}_{\circ}$ as usual. The $s$-convex functions in the second sense have been investigated [9].

Definition 1.7. [2] A set $K \subseteq \mathbb{R}^{n}$ is said to be invex with respect to the mapping $\eta: K \times K \longrightarrow \mathbb{R}^{n}$, if $x+$ t $\eta(y, x) \in K$ for every $x, y \in K$ and $t \in[0,1]$.

Notice that every convex set is invex with respect to the mapping $\eta(y, x)=y-x$, but the converse is not necessarily true $($ see $[[2],[34]])$ and the references therein.

Definition 1.8. [25] The function $f$ defined on the invex set $K \subseteq \mathbb{R}^{n}$ is said to be preinvex with respect $\eta$, if for every $x, y \in K$ and $t \in[0,1]$, we have that

$$
f(x+t \eta(y, x)) \leq(1-t) f(x)+t f(y)
$$

The concept of preinvexity is more general than convexity since every convex function is preinvex with respect to the mapping $\eta(y, x)=y-x$, but the converse is not true.

The Gauss-Jacobi type quadrature formula has the following

$$
\int_{a}^{b}(x-a)^{p}(b-x)^{q} f(x) d x=\sum_{k=0}^{+\infty} B_{m, k} f\left(\gamma_{k}\right)+R_{m}^{\star}|f|,
$$

for certain $B_{m, k}, \gamma_{k}$ and rest $R_{m}^{\star}|f|$, see [32].

Recently, Liu [21] obtained several integral inequalities for the left-hand side of (3) under the Definition 1.5 of $P$-function. Also Özdemir et al. in [24] established several integral inequalities concerning the left-hand side of (3) via some kinds of convexity.

Motivated by these results, in Section 2, the notion of new generalized $(s, m, \varphi)$-preinvex mapping is introduced and 
some new integral inequalities for the left-hand side of (3) involving new generalized $(s, m, \varphi)$-preinvex mappings along with beta function are given. In Section 3, some generalizations of Ostrowski type inequalities for new generalized $(s, m, \varphi)$-preinvex mappings via conformable fractional integrals are given. In Section 4, some applications to special means are given.

\section{New integral inequalities}

Definition 2.1. [7] A set $K \subseteq \mathbb{R}^{n}$ is said to be m-invex with respect to the mapping $\eta: K \times K \times(0,1] \longrightarrow \mathbb{R}^{n}$ for some fixed $m \in(0,1]$, if $m x+t \eta(y, m x) \in K$ holds for each $x, y \in K$ and any $t \in[0,1]$.

Remark 2.1. In Definition 2.1, under certain conditions, the mapping $\eta(y, m x)$ could reduce to $\eta(y, x)$. For example when $m=1$, then the $m$-invex set degenerates an invex set on $K$.

We next give new definition, to be referred as new generalized $(s, m, \varphi)$-preinvex mappings.

Definition 2.2. Let $K \subseteq \mathbb{R}$ be an open $m$-invex set with respect to $\eta: K \times K \longrightarrow \mathbb{R}$ and $\varphi: I \longrightarrow K$ a continuous function. For $f: K \longrightarrow \mathbb{R}$, any fixed $s \in(0,1]$ and some fixed $m \in(0,1]$, if

$$
f(m \varphi(x)+t \eta(\varphi(y), m \varphi(x))) \leq m(1-t)^{s} f(x)+t^{s} f(y)
$$

is valid for all $x, y \in I$ and $t \in[0,1]$, then we say that $f(x)$ is a new generalized $(s, m, \varphi)$-preinvex mapping with respect to $\eta$.

Remark 2.2. In Definition 2.2, it is worthwhile to note that the class of new generalized $(s, m, \varphi)$-preinvex mapping is a generalization of the class of s-convex in the second sense function given in Definition 1.6.

In this section, in order to prove our main results regarding some new integral inequalities involving new generalized $(s, m, \varphi)$-preinvex mappings along with beta function, we need the following new lemma.

Lemma 2.1. Let $\varphi: I \longrightarrow K$ be a continuous function. Assume that $f: K=[m \varphi(a), m \varphi(a)+\eta(\varphi(b), m \varphi(a))] \longrightarrow$ $\mathbb{R}$ is a continuous function on $K^{\circ}$ with respect to $\eta: K \times K \longrightarrow \mathbb{R}$, where $\eta(\varphi(b), m \varphi(a))>0$. Then for some fixed $m \in(0,1]$ and any fixed $p, q>0$, we have

$$
\begin{gathered}
\int_{m \varphi(a)}^{m \varphi(a)+\eta(\varphi(b), m \varphi(a))}(x-m \varphi(a))^{p}(m \varphi(a)+\eta(\varphi(b), m \varphi(a))-x)^{q} f(x) d x \\
=\eta^{p+q+1}(\varphi(b), m \varphi(a)) \int_{0}^{1} t^{p}(1-t)^{q} f(m \varphi(a)+t \eta(\varphi(b), m \varphi(a))) d t .
\end{gathered}
$$

Proof. It is easy to observe that

$$
\begin{gathered}
\int_{m \varphi(a)}^{m \varphi(a)+\eta(\varphi(b), m \varphi(a))}(x-m \varphi(a))^{p}(m \varphi(a)+\eta(\varphi(b), m \varphi(a))-x)^{q} f(x) d x \\
=\eta(\varphi(b), m \varphi(a)) \int_{0}^{1}(m \varphi(a)+t \eta(\varphi(b), m \varphi(a))-m \varphi(a))^{p} \\
\times(m \varphi(a)+\eta(\varphi(b), m \varphi(a))-m \varphi(a)-t \eta(\varphi(b), m \varphi(a)))^{q} \\
\times f(m \varphi(a)+t \eta(\varphi(b), m \varphi(a))) d t \\
=\eta^{p+q+1}(\varphi(b), m \varphi(a)) \int_{0}^{1} t^{p}(1-t)^{q} f(m \varphi(a)+t \eta(\varphi(b), m \varphi(a))) d t
\end{gathered}
$$

This completes the proof of the lemma.

Using Lemma 2.1, we now state the following theorems. 
Theorem 2.1. Let $\varphi: I \longrightarrow K$ be a continuous function. Assume that $f: K=[m \varphi(a), m \varphi(a)+\eta(\varphi(b), m \varphi(a))] \longrightarrow$ $\mathbb{R}$ is a continuous function on $K^{\circ}$ with $\eta(\varphi(b), m \varphi(a))>0$ and $k>1$. If $|f|^{\frac{k}{k-1}}$ is a new generalized $(s, m, \varphi)$-preinvex mapping on an open $m$-invex set $K$ with respect to $\eta: K \times K \longrightarrow \mathbb{R}$ for any fixed $s \in(0,1]$ and some fixed $m \in(0,1]$, then for any fixed $p, q>0$, we have

$$
\begin{aligned}
& \int_{m \varphi(a)}^{m \varphi(a)+\eta(\varphi(b), m \varphi(a))}(x-m \varphi(a))^{p}(m \varphi(a)+\eta(\varphi(b), m \varphi(a))-x)^{q} f(x) d x \\
\leq & \frac{\eta^{p+q+1}(\varphi(b), m \varphi(a))}{(s+1)^{\frac{k-1}{k}}}[\beta(k p+1, k q+1)]^{\frac{1}{k}}\left(m|f(a)|^{\frac{k}{k-1}}+|f(b)|^{\frac{k}{k-1}}\right)^{\frac{k-1}{k}} .
\end{aligned}
$$

Proof. Since $|f|^{\frac{k}{k-1}}$ is a new generalized $(s, m, \varphi)$-preinvex mapping on $K$, combining with Lemma 2.1, Hölder inequality and properties of the modulus, we get

$$
\begin{gathered}
\int_{m \varphi(a)}^{m \varphi(a)+\eta(\varphi(b), m \varphi(a))}(x-m \varphi(a))^{p}(m \varphi(a)+\eta(\varphi(b), m \varphi(a))-x)^{q} f(x) d x \\
\leq|\eta(\varphi(b), m \varphi(a))|^{p+q+1}\left[\int_{0}^{1} t^{k p}(1-t)^{k q} d t\right]^{\frac{1}{k}} \\
\times\left[\int_{0}^{1}|f(m \varphi(a)+t \eta(\varphi(b), m \varphi(a)))|^{\frac{k}{k-1}} d t\right]^{\frac{k-1}{k}} \\
\leq \eta^{p+q+1}(\varphi(b), m \varphi(a))[\beta(k p+1, k q+1)]^{\frac{1}{k}} \\
\times\left[\int_{0}^{1}\left(m(1-t)^{s}|f(a)|^{\frac{k}{k-1}}+t^{s}|f(b)|^{\frac{k}{k-1}}\right) d t\right]^{\frac{k-1}{k}} \\
=\frac{\eta^{p+q+1}(\varphi(b), m \varphi(a))}{(s+1)^{\frac{k-1}{k}}}[\beta(k p+1, k q+1)]^{\frac{1}{k}}\left(m|f(a)|^{\frac{k}{k-1}}+|f(b)|^{\frac{k}{k-1}}\right)^{\frac{k-1}{k}}
\end{gathered}
$$

So, the proof of this theorem is completed.

Theorem 2.2. Let $\varphi: I \longrightarrow K$ be a continuous function. Assume that $f: K=[m \varphi(a), m \varphi(a)+\eta(\varphi(b), m \varphi(a))] \longrightarrow$ $\mathbb{R}$ is a continuous function on $K^{\circ}$ with $\eta(\varphi(b), m \varphi(a))>0$ and $l \geq 1$. If $|f|^{l}$ is a new generalized $(s, m, \varphi)$-preinvex mapping on an open $m$-invex set $K$ with respect to $\eta: K \times K \longrightarrow \mathbb{R}$ for any fixed $s \in(0,1]$ and some fixed $m \in(0,1]$, then for any fixed $p, q>0$, we have

$$
\begin{gathered}
\int_{m \varphi(a)}^{m \varphi(a)+\eta(\varphi(b), m \varphi(a))}(x-m \varphi(a))^{p}(m \varphi(a)+\eta(\varphi(b), m \varphi(a))-x)^{q} f(x) d x \\
\leq \eta^{p+q+1}(\varphi(b), m \varphi(a))[\beta(p+1, q+1)]^{\frac{l-1}{l}} \\
\times\left[m|f(a)|^{l} \beta(p+1, q+s+1)+|f(b)|^{l} \beta(p+s+1, q+1)\right]^{\frac{1}{l}} .
\end{gathered}
$$

Proof. Since $|f|^{l}$ is a new generalized $(s, m, \varphi)$-preinvex mapping on $K$, combining with Lemma 2.1 , the well-known power mean inequality and properties of the modulus, we get

$$
\begin{gathered}
\int_{m \varphi(a)}^{m \varphi(a)+\eta(\varphi(b), m \varphi(a))}(x-m \varphi(a))^{p}(m \varphi(a)+\eta(\varphi(b), m \varphi(a))-x)^{q} f(x) d x \\
=\eta^{p+q+1}(\varphi(b), m \varphi(a)) \\
\times \int_{0}^{1}\left[t^{p}(1-t)^{q}\right]^{\frac{l-1}{l}}\left[t^{p}(1-t)^{q}\right]^{\frac{1}{l}} f(m \varphi(a)+t \eta(\varphi(b), m \varphi(a))) d t
\end{gathered}
$$




$$
\begin{gathered}
\leq|\eta(\varphi(b), m \varphi(a))|^{p+q+1}\left[\int_{0}^{1} t^{p}(1-t)^{q} d t\right]^{\frac{l-1}{l}} \\
\times\left[\int_{0}^{1} t^{p}(1-t)^{q}|f(m \varphi(a)+t \eta(\varphi(b), m \varphi(a)))|^{l} d t\right]^{\frac{1}{l}} \\
\leq \eta^{p+q+1}(\varphi(b), m \varphi(a))[\beta(p+1, q+1)]^{\frac{l-1}{l}} \\
\times\left[\int_{0}^{1} t^{p}(1-t)^{q}\left(m(1-t)^{s}|f(a)|^{l}+t^{s}|f(b)|^{l}\right) d t\right]^{\frac{1}{l}} \\
=\eta^{p+q+1}(\varphi(b), m \varphi(a))[\beta(p+1, q+1)]^{\frac{l-1}{l}} \\
\times\left[m|f(a)|^{l} \beta(p+1, q+s+1)+|f(b)|^{l} \beta(p+s+1, q+1)\right]^{\frac{1}{l}} .
\end{gathered}
$$

So, the proof of this theorem is completed.

\section{Ostrowski type conformable fractional integrals}

In this section, in order to prove our main results regarding some generalizations of Ostrowski type inequalities for new generalized $(s, m, \varphi)$-preinvex mappings via conformable fractional integrals, we need the following new lemma.

Lemma 3.1. Let $\varphi: I \longrightarrow K$ be a continuous function. Suppose $K=[m \varphi(a), m \varphi(a)+\eta(\varphi(b), m \varphi(a))] \subseteq \mathbb{R}$ be an open $m$-invex subset with respect to $\eta: K \times K \longrightarrow \mathbb{R}$ for some fixed $m \in(0,1]$ and let $\eta(\varphi(b), m \varphi(a))>0$. Assume that $f: K \longrightarrow \mathbb{R}$ is a differentiable function on $K^{\circ}$ and $f^{\prime} \in L_{1}(K)$. Then for $\alpha \in(n, n+1]$, where $n=0,1,2, \ldots$, we have

$$
\begin{gathered}
\frac{\beta(n+1, \alpha-n)}{\eta(\varphi(b), m \varphi(a))} \times\left\{\eta^{\alpha}(\varphi(x), m \varphi(a)) f(m \varphi(a)+\eta(\varphi(x), m \varphi(a)))\right. \\
\left.-\eta^{\alpha}(\varphi(x), m \varphi(b)) f(m \varphi(b)+\eta(\varphi(x), m \varphi(b)))\right\} \\
-\frac{n !}{\eta(\varphi(b), m \varphi(a))} \\
\times\left[\left({ }^{(m \varphi(a)+\eta(\varphi(x), m \varphi(a)))} I_{\alpha} f\right)(m \varphi(a))-\left({ }^{(m \varphi(b)+\eta(\varphi(x), m \varphi(b)))} I_{\alpha} f\right)(m \varphi(b))\right] \\
=\frac{\eta^{\alpha+1}(\varphi(x), m \varphi(a))}{\eta(\varphi(b), m \varphi(a))} \int_{0}^{1} \beta_{t}(n+1, \alpha-n) f^{\prime}(m \varphi(a)+t \eta(\varphi(x), m \varphi(a))) d t \\
-\frac{\eta^{\alpha+1}(\varphi(x), m \varphi(b))}{\eta(\varphi(b), m \varphi(a))} \int_{0}^{1} \beta_{t}(n+1, \alpha-n) f^{\prime}(m \varphi(b)+t \eta(\varphi(x), m \varphi(b))) d t .
\end{gathered}
$$

We denote

$$
\begin{gathered}
I_{f, \eta, \varphi}(x ; \alpha, n, m, a, b) \\
:=\frac{\eta^{\alpha+1}(\varphi(x), m \varphi(a))}{\eta(\varphi(b), m \varphi(a))} \int_{0}^{1} \beta_{t}(n+1, \alpha-n) f^{\prime}(m \varphi(a)+t \eta(\varphi(x), m \varphi(a))) d t \\
-\frac{\eta^{\alpha+1}(\varphi(x), m \varphi(b))}{\eta(\varphi(b), m \varphi(a))} \int_{0}^{1} \beta_{t}(n+1, \alpha-n) f^{\prime}(m \varphi(b)+t \eta(\varphi(x), m \varphi(b))) d t .
\end{gathered}
$$


Proof. Integrating by parts, we get

$$
\begin{aligned}
& I_{f, \eta, \varphi}(x ; \alpha, n, m, a, b) \\
& =\frac{\eta^{\alpha+1}(\varphi(x), m \varphi(a))}{\eta(\varphi(b), m \varphi(a))}\left[\left.\beta_{t}(n+1, \alpha-n) \frac{f(m \varphi(a)+t \eta(\varphi(x), m \varphi(a)))}{\eta(\varphi(x), m \varphi(a))}\right|_{0} ^{1}\right. \\
& \left.-\int_{0}^{1} \frac{t^{n}(1-t)^{\alpha-n-1} f(m \varphi(a)+t \eta(\varphi(x), m \varphi(a)))}{\eta(\varphi(x), m \varphi(a))} d t\right] \\
& -\frac{\eta^{\alpha+1}(\varphi(x), m \varphi(b))}{\eta(\varphi(b), m \varphi(a))}\left[\left.\beta_{t}(n+1, \alpha-n) \frac{f(m \varphi(b)+t \eta(\varphi(x), m \varphi(b)))}{\eta(\varphi(x), m \varphi(b))}\right|_{0} ^{1}\right. \\
& \left.-\int_{0}^{1} \frac{t^{n}(1-t)^{\alpha-n-1} f(m \varphi(b)+t \eta(\varphi(x), m \varphi(b)))}{\eta(\varphi(x), m \varphi(b))} d t\right] \\
& =\frac{\beta(n+1, \alpha-n)}{\eta(\varphi(b), m \varphi(a))} \\
& \times\left\{\eta^{\alpha}(\varphi(x), m \varphi(a)) f(m \varphi(a)+\eta(\varphi(x), m \varphi(a)))\right. \\
& \left.-\eta^{\alpha}(\varphi(x), m \varphi(b)) f(m \varphi(b)+\eta(\varphi(x), m \varphi(b)))\right\} \\
& -\frac{n !}{\eta(\varphi(b), m \varphi(a))} \\
& \times\left[\left((m \varphi(a)+\eta(\varphi(x), m \varphi(a))) I_{\alpha} f\right)(m \varphi(a))-\left((m \varphi(b)+\eta(\varphi(x), m \varphi(b))) I_{\alpha} f\right)(m \varphi(b))\right] .
\end{aligned}
$$

This completes the proof of the lemma.

Remark 3.1. If we choose $\alpha=n+1$ where $n=0,1,2, \ldots, m=1$ and $\eta(\varphi(y), \varphi(x))$ $=\varphi(y)-\varphi(x), \varphi(x)=x, \forall x, y \in I$ in Lemma 3.1, we get ([22], Lemma 1).

By using Lemma 3.1, the following results can be obtained for the corresponding version for power of first derivative.

Theorem 3.1. Let $\varphi: I \longrightarrow K$ be a continuous function. Suppose $K \subseteq \mathbb{R}$ be an open m-invex subset with respect to $\eta: K \times K \longrightarrow \mathbb{R}$ for any fixed $s \in(0,1]$, for some fixed $m \in(0,1]$ and let $\eta(\varphi(b), m \varphi(a))>0$. Assume that $f: K \longrightarrow \mathbb{R}$ is a differentiable function on $K^{\circ}$. If $\left|f^{\prime}\right|^{q}$ is a new generalized $(s, m, \varphi)$-preinvex mapping on $[m \varphi(a), m \varphi(a)+\eta(\varphi(b), m \varphi(a))], q>1, p^{-1}+q^{-1}=1$, then the following inequality for conformable fractional integrals holds:

$$
\begin{aligned}
& \left|I_{f, \eta, \varphi}(x ; \alpha, n, m, a, b)\right| \leq\left(\frac{1}{s+1}\right)^{\frac{1}{q}} \frac{\delta^{\frac{1}{p}}}{\eta(\varphi(b), m \varphi(a))} \\
& \quad \times\left\{|\eta(\varphi(x), m \varphi(a))|^{\alpha+1}\left[m\left|f^{\prime}(a)\right|^{q}+\left|f^{\prime}(x)\right|^{q}\right]^{\frac{1}{q}}\right. \\
& \left.\quad+|\eta(\varphi(x), m \varphi(b))|^{\alpha+1}\left[m\left|f^{\prime}(b)\right|^{q}+\left|f^{\prime}(x)\right|^{q}\right]^{\frac{1}{q}}\right\},
\end{aligned}
$$

where $\delta:=\int_{0}^{1} \beta_{t}^{p}(n+1, \alpha-n) d t$ and $\alpha \in(n, n+1], n=0,1,2, \ldots$ 
Proof. Since $\left|f^{\prime}\right|^{q}$ is a new generalized $(s, m, \varphi)$-preinvex mapping, combining with Lemma 3.1, Hölder inequality and taking the modulus, we have

$$
\begin{aligned}
& \left|I_{f, \eta, \varphi}(x ; \alpha, n, m, a, b)\right| \\
& \leq \frac{|\eta(\varphi(x), m \varphi(a))|^{\alpha+1}}{|\eta(\varphi(b), m \varphi(a))|} \int_{0}^{1} \beta_{t}(n+1, \alpha-n)\left|f^{\prime}(m \varphi(a)+t \eta(\varphi(x), m \varphi(a)))\right| d t \\
& +\frac{|\eta(\varphi(x), m \varphi(b))|^{\alpha+1}}{|\eta(\varphi(b), m \varphi(a))|} \int_{0}^{1} \beta_{t}(n+1, \alpha-n)\left|f^{\prime}(m \varphi(b)+t \eta(\varphi(x), m \varphi(b)))\right| d t \\
& \leq \frac{|\eta(\varphi(x), m \varphi(a))|^{\alpha+1}}{\eta(\varphi(b), m \varphi(a))}\left(\int_{0}^{1} \beta_{t}^{p}(n+1, \alpha-n) d t\right)^{\frac{1}{p}} \\
& \times\left(\int_{0}^{1}\left|f^{\prime}(m \varphi(a)+t \eta(\varphi(x), m \varphi(a)))\right|^{q} d t\right)^{\frac{1}{q}} \\
& +\frac{|\eta(\varphi(x), m \varphi(b))|^{\alpha+1}}{\eta(\varphi(b), m \varphi(a))}\left(\int_{0}^{1} \beta_{t}^{p}(n+1, \alpha-n) d t\right)^{\frac{1}{p}} \\
& \times\left(\int_{0}^{1}\left|f^{\prime}(m \varphi(b)+t \eta(\varphi(x), m \varphi(b)))\right|^{q} d t\right)^{\frac{1}{q}} \\
& \leq \frac{|\eta(\varphi(x), m \varphi(a))|^{\alpha+1}}{\eta(\varphi(b), m \varphi(a))}\left(\int_{0}^{1} \beta_{t}^{p}(n+1, \alpha-n) d t\right)^{\frac{1}{p}} \\
& \times\left[\int_{0}^{1}\left(m(1-t)^{s}\left|f^{\prime}(a)\right|^{q}+t^{s}\left|f^{\prime}(x)\right|^{q}\right) d t\right]^{\frac{1}{q}} \\
& +\frac{|\eta(\varphi(x), m \varphi(b))|^{\alpha+1}}{\eta(\varphi(b), m \varphi(a))}\left(\int_{0}^{1} \beta_{t}^{p}(n+1, \alpha-n) d t\right)^{\frac{1}{p}} \\
& \times\left[\int_{0}^{1}\left(m(1-t)^{s}\left|f^{\prime}(b)\right|^{q}+t^{s}\left|f^{\prime}(x)\right|^{q}\right) d t\right]^{\frac{1}{q}} \\
& =\left(\frac{1}{s+1}\right)^{\frac{1}{q}} \frac{\delta^{\frac{1}{p}}}{\eta(\varphi(b), m \varphi(a))} \\
& \times\left\{|\eta(\varphi(x), m \varphi(a))|^{\alpha+1}\left[m\left|f^{\prime}(a)\right|^{q}+\left|f^{\prime}(x)\right|^{q}\right]^{\frac{1}{q}}\right. \\
& \left.+|\eta(\varphi(x), m \varphi(b))|^{\alpha+1}\left[m\left|f^{\prime}(b)\right|^{q}+\left|f^{\prime}(x)\right|^{q}\right]^{\frac{1}{q}}\right\} .
\end{aligned}
$$

So, the proof of this theorem is completed.

Corollary 3.1. Under the same conditions as in Theorem 3.1, if we choose $\alpha=n+1, n=0,1,2, \ldots$, and $\left|f^{\prime}\right| \leq K$, we get the following inequality for fractional integrals:

$$
\begin{gathered}
\mid \frac{1}{\eta(\varphi(b), m \varphi(a))} \times\left\{\eta^{\alpha}(\varphi(x), m \varphi(a)) f(m \varphi(a)+\eta(\varphi(x), m \varphi(a)))\right. \\
\left.-\eta^{\alpha}(\varphi(x), m \varphi(b)) f(m \varphi(b)+\eta(\varphi(x), m \varphi(b)))\right\} \\
-\frac{\Gamma(\alpha+1)}{\eta(\varphi(b), m \varphi(a))} \\
\left.\times\left[J_{(m \varphi(a)+\eta(\varphi(x), m \varphi(a)))^{-}}^{\alpha} f(m \varphi(a))-J_{(m \varphi(b)+\eta(\varphi(x), m \varphi(b)))^{-}}^{\alpha} f(m \varphi(b))\right)\right]
\end{gathered}
$$




$$
\leq \frac{K}{(p \alpha+1)^{1 / p}}\left(\frac{m+1}{s+1}\right)^{\frac{1}{q}}\left[\frac{|\eta(\varphi(x), m \varphi(a))|^{\alpha+1}+|\eta(\varphi(x), m \varphi(b))|^{\alpha+1}}{\eta(\varphi(b), m \varphi(a))}\right] .
$$

Theorem 3.2. Let $\varphi: I \longrightarrow K$ be a continuous function. Suppose $K \subseteq \mathbb{R}$ be an open $m$-invex subset with respect to $\eta: K \times K \longrightarrow \mathbb{R}$ for any fixed $s \in(0,1]$, for some fixed $m \in(0,1]$ and let $\eta(\varphi(b), m \varphi(a))>0$. Assume that $f: K \longrightarrow \mathbb{R}$ is a differentiable function on $K^{\circ}$. If $\left|f^{\prime}\right|^{q}$ is a new generalized $(s, m, \varphi)$-preinvex mapping on $[m \varphi(a), m \varphi(a)+\eta(\varphi(b), m \varphi(a))], q \geq 1$, then the following inequality for conformable fractional integrals holds:

$$
\begin{aligned}
\left|I_{f, \eta, \varphi}(x ; \alpha, n, m, a, b)\right| \leq\left(\frac{1}{s+1}\right)^{\frac{1}{q}} \frac{(\beta(n+1, \alpha-n)-\beta(n+2, \alpha-n))^{1-\frac{1}{q}}}{\eta(\varphi(b), m \varphi(a))} \\
\times\left\{| \eta ( \varphi ( x ) , m \varphi ( a ) ) | ^ { \alpha + 1 } \left[m\left|f^{\prime}(a)\right|^{q} \beta(n+1, \alpha-n+s+1)\right.\right. \\
\left.\quad+\left|f^{\prime}(x)\right|^{q}(\beta(n+1, \alpha-n)-\beta(n+s+2, \alpha-n))\right]^{\frac{1}{q}} \\
+|\eta(\varphi(x), m \varphi(b))|^{\alpha+1}\left[m\left|f^{\prime}(b)\right|^{q} \beta(n+1, \alpha-n+s+1)\right. \\
\left.\left.+\left|f^{\prime}(x)\right|^{q}(\beta(n+1, \alpha-n)-\beta(n+s+2, \alpha-n))\right]^{\frac{1}{q}}\right\} .
\end{aligned}
$$

Proof. Since $\left|f^{\prime}\right|^{q}$ is a new generalized $(s, m, \varphi)$-preinvex mapping, combining with Lemma 3.1, the well-known power mean inequality and taking the modulus, we have

$$
\begin{aligned}
& \left|I_{f, \eta, \varphi}(x ; \alpha, n, m, a, b)\right| \\
& \leq \frac{|\eta(\varphi(x), m \varphi(a))|^{\alpha+1}}{|\eta(\varphi(b), m \varphi(a))|} \int_{0}^{1} \beta_{t}(n+1, \alpha-n)\left|f^{\prime}(m \varphi(a)+t \eta(\varphi(x), m \varphi(a)))\right| d t \\
& +\frac{|\eta(\varphi(x), m \varphi(b))|^{\alpha+1}}{|\eta(\varphi(b), m \varphi(a))|} \int_{0}^{1} \beta_{t}(n+1, \alpha-n)\left|f^{\prime}(m \varphi(b)+t \eta(\varphi(x), m \varphi(b)))\right| d t \\
& \leq \frac{|\eta(\varphi(x), m \varphi(a))|^{\alpha+1}}{\eta(\varphi(b), m \varphi(a))}\left(\int_{0}^{1} \beta_{t}(n+1, \alpha-n) d t\right)^{1-\frac{1}{q}} \\
& \times\left(\int_{0}^{1} \beta_{t}(n+1, \alpha-n)\left|f^{\prime}(m \varphi(a)+t \eta(\varphi(x), m \varphi(a)))\right|{ }^{q} d t\right)^{\frac{1}{q}} \\
& +\frac{|\eta(\varphi(x), m \varphi(b))|^{\alpha+1}}{\eta(\varphi(b), m \varphi(a))}\left(\int_{0}^{1} \beta_{t}(n+1, \alpha-n) d t\right)^{1-\frac{1}{q}} \\
& \times\left(\int_{0}^{1} \beta_{t}(n+1, \alpha-n)\left|f^{\prime}(m \varphi(b)+t \eta(\varphi(x), m \varphi(b)))\right|{ }^{q} d t\right)^{\frac{1}{q}} \\
& \leq \frac{|\eta(\varphi(x), m \varphi(a))|^{\alpha+1}}{\eta(\varphi(b), m \varphi(a))}\left(\int_{0}^{1} \beta_{t}(n+1, \alpha-n) d t\right)^{1-\frac{1}{q}} \\
& \times\left[\int_{0}^{1} \beta_{t}(n+1, \alpha-n)\left(m(1-t)^{s}\left|f^{\prime}(a)\right|^{q}+t^{s}\left|f^{\prime}(x)\right|^{q}\right) d t\right]^{\frac{1}{q}} \\
& +\frac{|\eta(\varphi(x), m \varphi(b))|^{\alpha+1}}{\eta(\varphi(b), m \varphi(a))}\left(\int_{0}^{1} \beta_{t}(n+1, \alpha-n) d t\right)^{1-\frac{1}{q}} \\
& \times\left[\int_{0}^{1} \beta_{t}(n+1, \alpha-n)\left(m(1-t)^{s}\left|f^{\prime}(b)\right|^{q}+t^{s}\left|f^{\prime}(x)\right|^{q}\right) d t\right]^{\frac{1}{q}}
\end{aligned}
$$




$$
\begin{aligned}
& =\left(\frac{1}{s+1}\right)^{\frac{1}{q}} \frac{(\beta(n+1, \alpha-n)-\beta(n+2, \alpha-n))^{1-\frac{1}{q}}}{\eta(\varphi(b), m \varphi(a))} \\
& \times\left\{| \eta ( \varphi ( x ) , m \varphi ( a ) ) | ^ { \alpha + 1 } \left[m\left|f^{\prime}(a)\right|^{q} \beta(n+1, \alpha-n+s+1)\right.\right. \\
& \left.\quad+\left|f^{\prime}(x)\right|^{q}(\beta(n+1, \alpha-n)-\beta(n+s+2, \alpha-n))\right]^{\frac{1}{q}} \\
& +|\eta(\varphi(x), m \varphi(b))|^{\alpha+1}\left[m\left|f^{\prime}(b)\right|^{q} \beta(n+1, \alpha-n+s+1)\right. \\
& \left.\left.+\left|f^{\prime}(x)\right|^{q}(\beta(n+1, \alpha-n)-\beta(n+s+2, \alpha-n))\right]^{\frac{1}{q}}\right\} .
\end{aligned}
$$

So, the proof of this theorem is completed.

Corollary 3.2. Under the same conditions as in Theorem 3.2, if we choose $\alpha=n+1, n=0,1,2, \ldots$, and $\left|f^{\prime}\right| \leq K$, we get the following inequality for fractional integrals:

$$
\begin{gathered}
\mid \frac{1}{\eta(\varphi(b), m \varphi(a))} \times\left\{\eta^{\alpha}(\varphi(x), m \varphi(a)) f(m \varphi(a)+\eta(\varphi(x), m \varphi(a)))\right. \\
\left.-\eta^{\alpha}(\varphi(x), m \varphi(b)) f(m \varphi(b)+\eta(\varphi(x), m \varphi(b)))\right\} \\
-\frac{\Gamma(\alpha+1)}{\eta(\varphi(b), m \varphi(a))} \\
\times\left[J_{(m \varphi(a)+\eta(\varphi(x), m \varphi(a)))^{-}}^{\alpha} f(m \varphi(a))-J_{(m \varphi(b)+\eta(\varphi(x), m \varphi(b)))^{\alpha}}^{\alpha} f(m \varphi(b))\right] \mid \\
\leq \frac{K}{(\alpha+1)^{1-\frac{1}{q}}}\left(m \beta(\alpha+1, s+1)+\frac{1}{\alpha+s+1}\right)^{\frac{1}{q}} \\
\times\left[\frac{|\eta(\varphi(x), m \varphi(a))|^{\alpha+1}+|\eta(\varphi(x), m \varphi(b))|^{\alpha+1}}{\eta(\varphi(b), m \varphi(a))}\right] .
\end{gathered}
$$

\section{Applications to special means}

Definition 4.1. [3] A function $M: \mathbb{R}_{+}^{2} \longrightarrow \mathbb{R}_{+}$, is called a Mean function if it has the following properties:

(1) Homogeneity: $M(a x, a y)=a M(x, y)$, for all $a>0$,

(2) Symmetry: $M(x, y)=M(y, x)$,

(3) Reflexivity: $M(x, x)=x$,

(4) Monotonicity: If $x \leq x^{\prime}$ and $y \leq y^{\prime}$, then $M(x, y) \leq M\left(x^{\prime}, y^{\prime}\right)$,

(5) Internality: $\min \{x, y\} \leq M(x, y) \leq \max \{x, y\}$.

We consider some means for arbitrary positive real numbers $\alpha, \beta(\alpha \neq \beta)$.

(1) The arithmetic mean:

$$
A:=A(\alpha, \beta)=\frac{\alpha+\beta}{2} .
$$

(2) The geometric mean:

$$
G:=G(\alpha, \beta)=\sqrt{\alpha \beta} .
$$

(3) The harmonic mean:

$$
H:=H(\alpha, \beta)=\frac{2}{\frac{1}{\alpha}+\frac{1}{\beta}} .
$$


(4) The power mean:

$$
P_{r}:=P_{r}(\alpha, \beta)=\left(\frac{\alpha^{r}+\beta^{r}}{2}\right)^{\frac{1}{r}}, r \geq 1
$$

(5) The identric mean:

$$
I:=I(\alpha, \beta)= \begin{cases}\frac{1}{e}\left(\frac{\beta^{\beta}}{\alpha^{\alpha}}\right), & \alpha \neq \beta \\ \alpha, & \alpha=\beta\end{cases}
$$

(6) The logarithmic mean:

$$
L:=L(\alpha, \beta)=\frac{\beta-\alpha}{\ln (\beta)-\ln (\alpha)}
$$

(7) The generalized log-mean:

$$
L_{p}:=L_{p}(\alpha, \beta)=\left[\frac{\beta^{p+1}-\alpha^{p+1}}{(p+1)(\beta-\alpha)}\right]^{\frac{1}{p}} ; p \in \mathbb{R} \backslash\{-1,0\} .
$$

(8) The weighted $p$-power mean:

$$
M_{p}\left(\begin{array}{cccc}
\alpha_{1}, & \alpha_{2}, & \cdots & , \alpha_{n} \\
u_{1}, & u_{2}, & \cdots & , u_{n}
\end{array}\right)=\left(\sum_{i=1}^{n} \alpha_{i} u_{i}^{p}\right)^{\frac{1}{p}}
$$

where $0 \leq \alpha_{i} \leq 1, u_{i}>0(i=1,2, \ldots, n)$ with $\sum_{i=1}^{n} \alpha_{i}=1$.

It is well known that $L_{p}$ is monotonic nondecreasing over $p \in \mathbb{R}$ with $L_{-1}:=L$ and $L_{0}:=I$. In particular, we have the following inequality $H \leq G \leq L \leq I \leq A$. Now, let $a$ and $b$ be positive real numbers such that $a<b$ and $\alpha \in(n, n+1]$, where $n=0,1,2, \ldots$ Consider the function $M:=M(\varphi(a), \varphi(b)):[\varphi(a), \varphi(a)+\eta(\varphi(b), \varphi(a))] \times$ $[\varphi(a), \varphi(a)+\eta(\varphi(b), \varphi(a))] \longrightarrow \mathbb{R}_{+}$, which is one of the above mentioned means and $\varphi: I \longrightarrow K$ be a continuous function. Therefore one can obtain various inequalities using the results of Section 3 for these means as follows. Replace $\eta(\varphi(y), m \varphi(x))$ with $\eta(\varphi(y), \varphi(x))$ and setting $\eta(\varphi(y), \varphi(x))=M(\varphi(x), \varphi(y)), \forall x, y \in I$ for value $m=1$ in (6) and (8), one can obtain the following interesting inequalities involving means:

$$
\begin{aligned}
& \left|I_{f, M(\cdot, \cdot), \varphi}(x ; \alpha, n, 1, a, b)\right| \\
& =\mid \frac{\beta(n+1, \alpha-n)}{M(\varphi(a), \varphi(b))} \times\left\{M^{\alpha}(\varphi(a), \varphi(x)) f(\varphi(a)+M(\varphi(a), \varphi(x)))\right. \\
& \left.-M^{\alpha}(\varphi(b), \varphi(x)) f(\varphi(b)+M(\varphi(b), \varphi(x)))\right\} \\
& -\frac{n !}{M(\varphi(a), \varphi(b))} \\
& \times\left[\left((\varphi(a)+M(\varphi(a), \varphi(x))) I_{\alpha} f\right)(\varphi(a))-\left((\varphi(b)+M(\varphi(b), \varphi(x))) I_{\alpha} f\right)(\varphi(b))\right] \mid \\
& \leq\left(\frac{1}{s+1}\right)^{\frac{1}{q}} \frac{\delta^{\frac{1}{p}}}{M(\varphi(a), \varphi(b))} \\
& \times\left\{M^{\alpha+1}(\varphi(a), \varphi(x))\left[\left|f^{\prime}(a)\right|^{q}+\left|f^{\prime}(x)\right|^{q}\right]^{\frac{1}{q}}\right. \\
& \left.+M^{\alpha+1}(\varphi(b), \varphi(x))\left[\left|f^{\prime}(b)\right|^{q}+\left|f^{\prime}(x)\right|^{q}\right]^{\frac{1}{q}}\right\} \\
& \left|I_{f, M(\cdot, \cdot), \varphi}(x ; \alpha, n, 1, a, b)\right| \leq\left(\frac{1}{s+1}\right)^{\frac{1}{q}} \frac{(\beta(n+1, \alpha-n)-\beta(n+2, \alpha-n))^{1-\frac{1}{q}}}{M(\varphi(a), \varphi(b))}
\end{aligned}
$$




$$
\begin{aligned}
& \times\left\{M ^ { \alpha + 1 } ( \varphi ( a ) , \varphi ( x ) ) \left[\left|f^{\prime}(a)\right|^{q} \beta(n+1, \alpha-n+s+1)\right.\right. \\
& \left.+\left|f^{\prime}(x)\right|^{q}(\beta(n+1, \alpha-n)-\beta(n+s+2, \alpha-n))\right]^{\frac{1}{q}} \\
& +M^{\alpha+1}(\varphi(b), \varphi(x))\left[\left|f^{\prime}(b)\right|^{q} \beta(n+1, \alpha-n+s+1)\right. \\
& \left.\left.+\left|f^{\prime}(x)\right|^{q}(\beta(n+1, \alpha-n)-\beta(n+s+2, \alpha-n))\right]^{\frac{1}{q}}\right\} .
\end{aligned}
$$

Letting $M(\varphi(x), \varphi(y)):=A, G, H, P_{r}, I, L, L_{p}, M_{p}, \forall x, y \in I$ in (10) and (11), we get the inequalities involving means for a particular choices of a differentiable new generalized $(s, 1, \varphi)$-preinvex mapping $f$. The details are left to the interested reader.

\section{References}

[1] T. Abdeljawad, On conformable fractional calculus, J. Comput. Appl. Math., 279, (2015), 57-66.

[2] T. Antczak, Mean value in invexity analysis, Nonlinear Anal., 60, (2005), 1473-1484.

[3] P. S. Bullen, Handbook of Means and Their Inequalities, Kluwer Academic Publishers, Dordrecht, (2003).

[4] Y.-M. Chu, M. Adil Khan, T. Ali and S. S. Dragomir, Inequalities for $\alpha$-fractional differentiable functions, $J$. Inequal. Appl., (2017) 2017:93 DOI10.1186/s13660-017-1371-6.

[5] S. S. Dragomir, Inequalities of Hermite-Hadamard type, Moroccan J. Pure Appl. Anal., 1(1), (2015), 1-21.

[6] S. S. Dragomir, J. Pečarić and L. E. Persson, Some inequalities of Hadamard type, Soochow J. Math., 21, (1995), 335-341.

[7] T. S. Du, J. G. Liao and Y. J. Li, Properties and integral inequalities of Hadamard-Simpson type for the generalized (s,m)-preinvex functions, J. Nonlinear Sci. Appl., 9, (2016), 3112-3126.

[8] S. Erden and M. Z. Sarikaya, New Hermite-Hadamard type inequalities for twice differentiable convex mappings via Green function and applications, Moroccan J. Pure Appl. Anal., 2(2), (2016), 107-117.

[9] H. Hudzik, L. Maligranda, Some remarks on s-convex functions, Aequationes Math., 48, (1994), 100-111.

[10] I. Işcan and S. Turhan, Generalized Hermite-Hadamard-Fejér type inequalities for GA-convex functions via fractional integral, Moroccan J. Pure Appl. Anal., 2(1), (2016), 34-46.

[11] A. Kashuri and R. Liko, Hermite-Hadamard type integral inequalities for products of two generalized $(s, m, \xi)$ preinvex functions, Moroccan J. Pure Appl. Anal., 3(1), (2017), 102-115.

[12] M. Adil Khan, Y. Khurshid, T. Ali and N. Rehman, Inequalities for three times differentiable functions, $J$. Math., Punjab Univ., 48(2), (2016), 35-48.

[13] M. Adil Khan, Y. Khurshid and T. Ali, Hermite-Hadamard inequality for fractional integrals via $\eta$-convex functions, Acta Math. Univ. Comenianae, 86(1), (2017), 153-164.

[14] M. Adil Khan, T. Ali, S. S. Dragomir and M. Z. Sarikaya, HermiteHadamard type inequalities for conformable fractional integrals, RACSAM, Rev. R. Acad. Cienc. Exactas Fs. Nat., Ser. A Mat., 2017(2): 1-16 DOI10.1007/s13398-017-0408-5.

[15] M. Adil Khan, Y.-M. Chu, T. U. Khan and J. Khan, Some new inequalities of Hermite-Hadamard type for s-convex functions with applications, Open Math., 15, (2017), 1414-1430.

[16] M. Adil Khan, S. Begum, Y. Khurshid and Y.-M. Chu, Ostrowski type inequalities involving conformable fractional integrals, J. Inequal. Appl., 2018(70), (2018), 1-14.

[17] M. Adil Khan, Y.-M. Chu, A. Kashuri and R. Liko, Hermite-Hadamard type fractional integral inequalities for $M T_{(r ; g, m, \varphi)}$-preinvex functions, J. Comput. Anal. Appl., 26(8), (2019), 1487-1503.

[18] M. Adil Khan, Y.-M. Chu, A. Kashuri, R. Liko and G. Ali, New Hermite-Hadamard inequalities for conformable fractional integrals, J. Funct. Spaces, In press.

[19] R. Khalil, M. Al Horani, A. Yousef and M. Sababheh, A new definition of fractional derivative, J. Comput. Appl. Math., 264, (2014), 65-70. 
[20] M. Kunt and I. Işcan, Hermite-Hadamard type inequalities for $p$-convex functions via fractional integrals, Moroccan J. Pure Appl. Anal., 3(1), (2017), 22-35.

[21] W. Liu, New integral inequalities involving beta function via P-convexity, Miskolc Math. Notes, 15(2), (2014), 585-591.

[22] W. Liu, W. Wen and J. Park, Ostrowski type fractional integral inequalities for MT-convex functions, Miskolc Math. Notes, 16(1), (2015), 249-256.

[23] W. Liu, W. Wen and J. Park, Hermite-Hadamard type inequalities for MT-convex functions via classical integrals and fractional integrals, J. Nonlinear Sci. Appl., 9, (2016), 766-777.

[24] M. E. Özdemir, E. Set and M. Alomari, Integral inequalities via several kinds of convexity, Creat. Math. Inform., 20(1), (2011), 62-73.

[25] R. Pini, Invexity and generalized convexity, Optimization, 22, (1991), 513-525.

[26] E. Set and A. Gözpinar, A study on Hermite-Hadamard type inequalities for $s$-convex functions via conformable fractional integrals, Submitted.

[27] E. Set and I. Mumcu, Hermite-Hadamard-Fejér type inequalies for conformable fractional integrals, (Submitted).

[28] E. Set, A. O. Akdemir and I. Mumcu, Ostrowski type inequalities for functions whose derivatives are convex via conformable fractional integrals, Submitted.

[29] E. Set, A. O. Akdemir and I. Mumcu, Chebyshev type inequalities for conformable fractional integrals, Submitted.

[30] E. Set, M. E. Özdemir, M. Z. Sarikaya and F. Karakoç, Hermite-Hadamard type inequalities for $(\alpha, m)$-convex functions via fractional integrals, Moroccan J. Pure Appl. Anal., 3(1), (2017), 15-21.

[31] E. Set, M. Z. Sarikaya and A. Gözpinar, Some Hermite-Hadamard type inequalities for convex functions via conformable fractional integrals and related inequalities, Creat. Math. Inform., Accepted paper.

[32] D. D. Stancu, G. Coman and P. Blaga, Analiză numerică şi teoria aproximării, Cluj-Napoca: Presa Universitară Clujeană., 2, (2002).

[33] M. Tunç, Ostrowski type inequalities for functions whose derivatives are MT-convex, J. Comput. Anal. Appl., 17(4), (2014), 691-696.

[34] X. M. Yang, X. Q. Yang and K. L. Teo, Generalized invexity and generalized invariant monotonicity, J. Optim. Theory Appl., 117, (2003), 607-625.

[35] Y. Zhang, T. S. Du, H. Wang, Y.-J. Shen and A. Kashuri, Extensions of different type parameterized inequalities for generalized $(m, h)$-preinvex mappings via $k$-fractional integrals, J. Inequal. Appl., 2018(49), (2018), 1-30. 